\title{
Trimming of Silicon Nitride Microring Resonators with a Polysilane Top Cladding
}

\section{Daniel K. Sparacin, John P. Lock, Ching-yin Hong, K. K. Gleason, L. C. Kimerling and Jurgen Michel} Massachusetts Institute of Technology, 77 Massachusetts Avenue, Cambridge, MA 02139

We demonstrate $23.5 \mathrm{~nm}$ shifts of $\mathrm{Si}_{3} \mathrm{~N}_{4}$ microring resonances by exposing a polysilane cladding layer to UV light. Our PECVD polysilane cladding is superior to other approaches, because it is insoluble, demonstrates good stability, and exhibits $\sim 4 \%$ index shift.

Microring resonators are a basic building block of photonic circuits, enabling complex functionality for optical systems. Such resonators can serve as filters for wavelength division multiplexing and demultiplexing of broadband optical signals [1], dispersion compensators for accurately controlling phase [2], lasers [3], and ultrafast all-optical switches [4]. The resonance condition for a ring resonator relates $\mathrm{D}$, the diameter of the ring; $\lambda_{0}$, the free space wavelength of resonant light; $\mathrm{m}$, an integer indicating the resonator mode number; and $\mathrm{n}$, the effective index of the ring (Eq. 1).

$$
\lambda_{o}=n\left(\frac{\pi D}{m}\right)
$$

Precise control over $\lambda_{0}$ in each ring is critical for microphotonics integration. As ring diameters shrink to less than $10 \mu \mathrm{m}$, non-deterministic pattern transfer errors limit dimensional precision and cause $\lambda_{0}$ to shift by several nanometers for identical devices. Thus, a postproduction trimming process to modify $\mathrm{n}$ and control $\lambda_{0}$ is essential. Assuming single mode operation and continuous resonator mode number, the predicted shift of the resonance $\Delta \lambda$ can be derived from Eq. 1, where the subscripts 1 and 2 indicate initial and final effective indices.

$$
\Delta \lambda=\lambda_{0,1}\left(\frac{n_{2}}{n_{1}}-1\right)
$$

Conventional trimming methods utilize resistive micro-heaters to induce a thermo-optic response in the core material, where the small magnitude of these thermo-optic coefficients $\left(10^{-5}-10^{-4} \mathrm{~K}^{-1}\right.$ for most dielectrics) corresponds to a feasible thermal trimming range of several nanometers. This architecture adds several steps to the fabrication of a photonic circuit, limits the density of devices to maintain thermal isolation, and requires significant power consumption to keep the rings "trimmed".

An alternative trimming method utilizes a polysilane polymer film as the cladding material and adjusts its refractive index via photo-oxidation when irradiated with UV light [6]. Having a refractive index similar to silicon dioxide $\left(\mathrm{SiO}_{2}\right)$, the predominant material of choice for cladding layers, polysilane polymers can be integrated with $\mathrm{Si}, \mathrm{SiON}$, and $\mathrm{Si}_{3} \mathrm{~N}_{4}$ high index-contrast microring resonators. The material is transmissive over a broad range of visible and near-IR light $[7,8]$ useful for microphotonics applications [9].

We demonstrate a vapor-phase technique for depositing an amorphous and highly cross-linked polysilane (PECVD 6M2S) top cladding layer [10] directly onto ring resonator devices. PECVD $6 \mathrm{M} 2 \mathrm{~S}$ is insoluble (unlike dip-coated materials), does not swell in organic solvents, and demonstrates good stability in ambient light, atmosphere, and temperature. Photo- 
oxidation trimming of PECVD 6M2S cladding provides a precise, localized, controllable, and micro-fab compatible technique for specifying the resonance condition of ring resonators.

\section{Experiment}

$\mathrm{Si}_{3} \mathrm{~N}_{4}$ microring resonators, designed for single mode operation at $\lambda=1550 \mathrm{~nm}$, were fabricated on a 3 $\mu \mathrm{m} \mathrm{SiO}_{2}$ under-cladding layer on (100) $\mathrm{Si}$. The microrings have a diameter of $100 \mu \mathrm{m}$ and have crosssectional dimensions of $400 \times 750 \mathrm{~nm}^{2}$. Next, a $1 \mu \mathrm{m}$ thick PECVD 6M2S top cladding layer was deposited directly onto the ring resonators. Spectral characterization of the microrings was performed for the TE and TM polarizations with a JDS Uniphase tunable laser and broadband photodetector used in conjunction with a Newport Auto-Align System. A MINERALIGHT ${ }^{\circledR}$ handheld lamp (model UVGL-25) emitting $\lambda=254 \mathrm{~nm}$ UV light was used to irradiate samples with a flux of $1.7 \mu \mathrm{W} / \mathrm{cm}^{2}$.

An identical UV irradiation process was conducted separately on a sample from the same wafer and the PECVD 6M2S film was measured after each exposure using a Woolam M-2000 variable angle spectroscopic ellipsometer (VASE). Ellipsometry data was fit to the Cauchy-Urbach model yielding the film thickness and refractive index at $1550 \mathrm{~nm}$.

\section{Discussion}

A controllable decrease in the refractive index of the PECVD 6M2S cladding layer was achieved using photooxidation induced by UV irradiation (Fig. 1). The refractive index decreased by $\sim 4 \%$, from 1.52 to 1.46 at $\lambda=1550 \mathrm{~nm}$ and was exponential in nature. UV light having a wavelength less than $300 \mathrm{~nm}$ causes chain scission in polysilane polymeric materials, and subsequent oxidation converts $\mathrm{Si}-\mathrm{Si}$ bonds into $\mathrm{Si}-\mathrm{O}-\mathrm{Si}$ bonds [12] (Fig. 1 inset). The resulting index change is about $50 \%$ greater than the response observed with a previously reported dip-coated polysilane material [6].

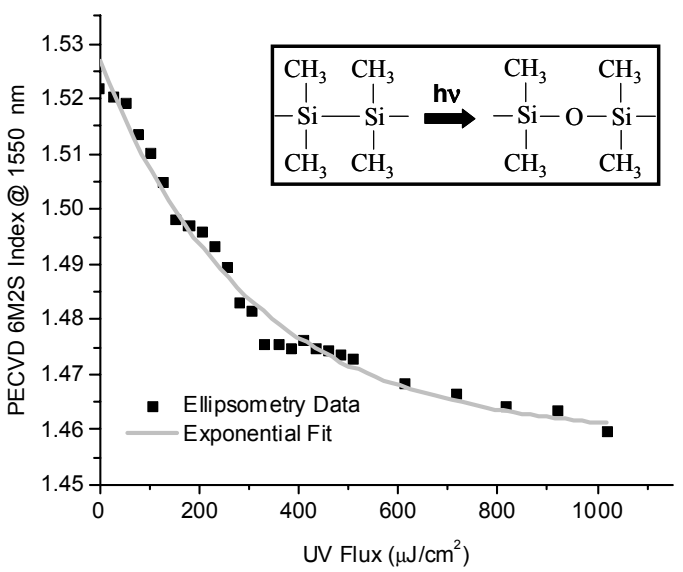

Figure 1: The refractive index of PECVD 6M2S versus UV flux.

To compare experiment with theory, a model was formulated using the relation found in Eq. 2 and the exposure curve data in Fig. 1. The resulting theoretical resonance shifts (Fig. 2) agree well with the experimental data in terms of functional form, magnitude, and are continuous with irradiation.

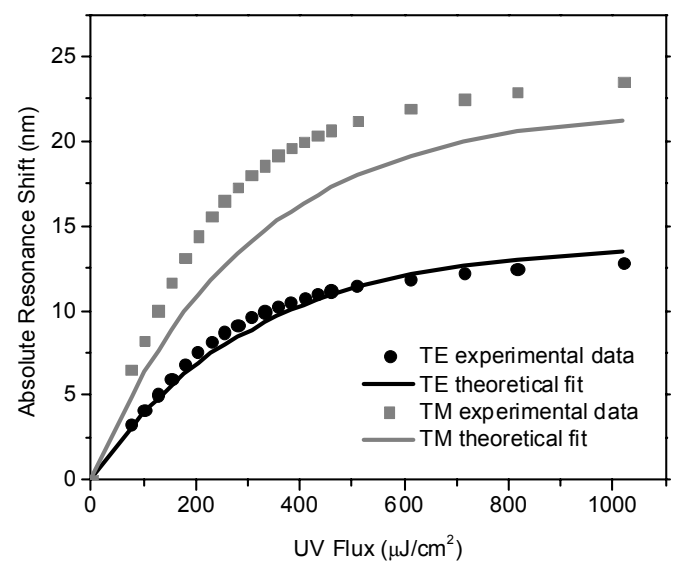

Figure 2: Experimental and theoretical resonance shifts of the $\mathrm{Si}_{3} \mathrm{~N}_{4}$ ring Resonator for the TE and $\mathrm{TM}$ polarizations.

The overall resonance shifts, after a UV flux of 1000 $\mu \mathrm{J} / \mathrm{cm}^{2}$, were $12.8 \mathrm{~nm}$ for the TE mode and $23.5 \mathrm{~nm}$ for the TM mode (Fig. 2), exceeding the free spectral range (FSR) for both the TE $(3.9 \mathrm{~nm})$ and TM (4.5 nm) polarizations. These shifts are an order of magnitude 
greater than what can be obtained with thermal trimming for $\mathrm{Si}_{3} \mathrm{~N}_{4}$ waveguides.

An additional benefit of photo-oxidation trimming is the ability to focus UV light and thereby localize trimming to specified areas of a chip. This enables one to preserve the spectral response of higher order filters that require multiple rings. Differences between ring-toring and ring-to-bus coupling require localized index trimming on separate areas of the filter to keep all rings in resonance with each other.

Spectral responses of the trimmed ring resonators were also measured over a range of temperatures from 25 to $70^{\circ} \mathrm{C}$ (Fig. 3). The thermo-optic coefficient $(\mathrm{dn} / \mathrm{dT})$ for the system was found to be $-1.3 \times 10^{-4} \mathrm{~K}^{-1}$ for the TM mode. This value is an order of magnitude greater than $\mathrm{SiO}_{2}$ clad $\mathrm{Si}_{3} \mathrm{~N}_{4}$ ring resonators and enhances the potential thermal trimming/tuning range. Thermal hysteresis effects were not detected over this temperature range.

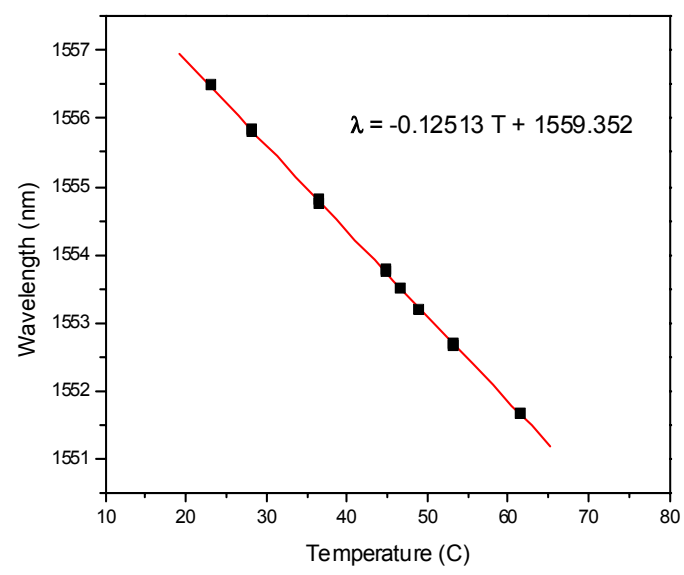

Figure 3 - Resonant wavelength versus temperature

\section{Conclusion}

PECVD 6M2S is a highly crosslinked and insoluble polymeric material with a refractive index that can decrease through photo-oxidation by nearly $4 \%$. This index change is $50 \%$ more than what has been shown using a soluble, dip-coated polysilane cladding layer.
Photo-oxidation trimming of PECVD 6M2S top cladding provides a precise, localized, and controllable technique for specifying the resonance condition of ring resonators.

\section{Acknowledgments}

This work was supported in part by the MRSEC Program of the National Science Foundation under Contract No. DMR 02-13282 and the U.S. Army through the Institute for Soldier Nanotechnologies, under Contract DAAD-19-02-D-0002 with the U.S. Army Research Office. The content does not necessarily reflect the position of the Government, and no official endorsement should be inferred.

\section{References}

1. B.E. Little, S.T.Chu, H.A.Haus, J.Foresi, J.-P.Laine, IEEE J. Lightwave Tech. 15, 998 (1997)

2. C.K. Madsen, G. Lenz, A.J. Bruce, M.A. Capuzzo, L.T. Gomez, T.N. Nielsen, I. Brener, Optics Letters, 24, No.22, 1555 (1999)

3. S.J. Choi, Z. Peng, Q. Yang, S.J. Choi, P.D. Dapkus, IEEE Phot. Tech. Lett., 16, 356 (2004)

4. V.R. Almeida, C.A. Barrios, R.R. Panepucci, M. Lipson, Nature, 43, 1081 (2004)

5. P. Rabiei, W.H. Steier, IEEE Phot. Tech. Lett., 15, 1255 (2003)

6. S.T.Chu, W.Pan, S.Sato, T.Kaneko, B.E.Little, Y.Kokubun, IEEE Phot. Tech. Lett, 11, 688 (1999)

7. L. A. Hornak, T. W. Wedman, E. W. Kwock, J Apl Phys 67, 2235 (1990).

8. P. K. Tien, G. Smolinsky, R. J. Martin, Appl Optics 11, 637 (1972).

9. L. Eldada, "Polymer microphotonics," in Nano- and Micro-Optics for Information Systems, L. Eldada, ed., Proc SPIE 5225, 49 (2003).

10. J.P. Lock and K.K. Gleason, Appl Optics 44, 1691 (2005).

11. H.G. Tomkins and W.A. McGahan, Spectroscopic Ellipsometry and Reflectometry, Wiley-Interscience, New York (1999).

12. R.D. Miller and J. Michl, Chem Rev 89, 1359 (1989) 